\title{
On the origin of optical properties of natural organic matter: the role of excitation energy transfer and its experimental observation
}

Yakimov B.P. ${ }^{1}$, Rubekina A.A. ${ }^{1}$, Zherebker A.Ya. $^{1,2}$, Perminova I.V. ${ }^{1}$, Shirshin E.A. ${ }^{1}$

${ }^{1}$ Lomonosov Moscow State University, Moscow, Russia, shirshin@lid.phys.msu.ru

${ }^{2}$ Skolkovo Institute of Science and Technology, Moscow, Russia

doi: 10.36291/HIT.2019.yakimov.051

Despite being extensively studied for decades, the origin of natural organic matter (NOM) optical properties remains debatable. While the possibility to describe as a superposition of individual chromophores seems the most obvious approach, several facts contradict this hypothesis. As a result, speculations on the dominating role of chromophores interaction in the formation of NOM spectral properties are widely discussed in the literature. However, although it is quite obvious that electronic interaction must occur in such a complex molecular ensemble as NOM, a direct experimental study of this phenomenon has not been reported yet. In this work we aim at demonstration of the role of electronic interaction in the formation of NOM optical properties. Making use of time-resolved spectroscopy techniques in the subpicosecond to nanosecond ranges, we uncover the chain of events which lead to the formation of the red edge in NOM optical spectra. Then, we burn a hole in the NOM spectra and show how this affects the energy transfer chain. Based on these experiments, a semi-quantitative model of photophysical processes in NOM was developed.

Acknowledgements. Dr. E. Shirshin acknowledges the financial support of IHSS for the Young Investigator Research Grant in 2018. 